\title{
Palma y González Prada. Crónica de un distanciamiento
}

\author{
Alberto Varillas Montenegro \\ Academia Peruana de la Lengua \\ varillas.alberto@gmail.com \\ Lima-Perú
}

\section{RESUMEN}

Es bien sabido que durante los últimos años del siglo XIX Ricardo Palma fue víctima de duros y continuos ataques de Manuel González Prada y sus seguidores, atrincherados en el Club Literario. Desde hace más de un siglo, los estudiosos de la literatura de aquellos años han ensayado múltiples interpretaciones para explicar las razones que movieron a escritores y periodistas, de preferencia jóvenes, a atacar con tanta saña al autor de las Tradiciones Peruanas. Este trabajo se propone considerar el asunto desde una perspectiva generacional, lo cual no tiene por qué extrañar, pero se insiste en que el censurable nivel al cual llegaron los insultos se debe a la presencia en la Revista Social y en el Círculo Literario del periodista político y agudo escritor satírico Luis Enrique Márquez que una docena de años antes había atacado ferozmente a Palma en defensa del gobierno del presidente Manuel Pardo.

Palabras clave: Ricardo Palma, Manuel González Prada, Luis Enrique Márquez, Círculo Literario, Ateneo de Lima, confrontaciones políticas peruanas a fines del siglo XIX.

\section{Abstract}

It is well known that during the last years of the 19th century, Ricardo Palma was the victim of harsh and continuous attacks coming from Manuel González Prada and his followers, members of the Circulo Literario. For more than a century, literature researchers of those years have rehearsed multiple interpretations to explain the reasons that inspired writers and journalists, preferably young ones, to attack the author of Peruvian Traditions with such fury. The present article proposes to discuss the issue from a generational perspective, which is not surprising, but it insists that the censurable level the insults reached was due to the presence of 
the political journalist and satirist Luis Enrique Márquez in Revista Social and Círculo Literario, who a dozen years earlier had fiercely attacked Palma in defense of the government of President Manuel Pardo.

Keywords: Ricardo Palma, Manuel González Prada, Luis Enrique Márquez, Circulo Literario, Ateneo de Lima, Peruvian political confrontations in late 19th Century.

\section{Alberto Varillas Montenegro (Perú)}

Miembro de número de la Academia Peruana de la Lengua (Vicepresidente), la Academia Nacional de la Historia, el Instituto Riva Agüero y el Instituto Ricardo Palma. Se ha especializado en la historia y la literatura del Perú del siglo xix. Entre otras obras ha publicado Felipe Pardo y Aliaga, La literatura del Perú del siglo xix, Perú y Ecuador. Visión actual de un antiguo conflicto, Periodismo e historia del Perú en el siglo xix, Apuntes para una historiografía de la literatura peruana del siglo xix. Ha editado las Obras completas de Manuel A. Segura. 
Mucho se ha escrito sobre la relación que existió entre Ricardo Palma y Manuel González Prada entre los años que siguen a la guerra del Pacífico y el fallecimiento de ambos escritores, más de treinta años después. Menos se ha estudiado la vinculación que tuvieron estos importantes escritores peruanos en los años previos. Pasado con largueza un siglo de la muerte de ambos, bien vale la pena formular algunas consideraciones sobre la relación que hubo entre ellos durante aquellos años, cuando los dos compartían la vida peruana desde perspectivas etarias, sociales y económicas distintas.

Palma era once años mayor que González Prada pues ya ha quedado establecido que don Manuel nace en 1844 y no en 1848, tal como se repitió durante casi un siglo. Sin embargo, los dos escritores pertenecían a generaciones diferentes, Palma a la Romántica y González Prada a la Ecléctica, según he explicado en un trabajo anterior (Varillas, 1992). Por ello, bien vale la pena que nos preguntemos sobre sus años mozos, sobre la relación que pudo haber habido entre ellos y los posibles antecedentes de sus discrepancias finales.

\section{Los años mozos de dos escritores importantes}

El futuro tradicionista, hijo de padres provincianos, nace a principios de 1833 en una finca ubicada en el centro de Lima, muy cerca del Tribunal de la Inquisición, del convento de San Francisco y del monasterio de la Concepción, tres locales importantes en la capital de la nueva república. En el mismo año se cumplían solo siete de la capitulación del último reducto de las fuerzas armadas españolas en el Perú, el que sostenía el general Rodil en la fortaleza del Real Felipe en el Callao. Fresco estaba, pues, el recuerdo del coloniaje. Los viajeros que por aquellos años pasaban por Lima no la favorecen con sus recuerdos: carecía de veredas en las calles por las que las acequias 
centrales corrían libremente; la inseguridad ciudadana era muy grande, tanto de día cuanto de noche; era por entonces una ciudad oscura a la que el general Gamarra acababa de disponer la colocación de pescantes para colgar faroles con velas. Y en medio de esta ciudad, libre por la voluntad de su pueblo, pero empobrecida por la imperiosa necesidad de sostener guerras y debelar insurrecciones, el niño Manuel Ricardo Palma paseaba su curiosidad con mirada observadora y, cruzando por alguna de las portadas que adornaban sus murallas, llegaba hasta la Alameda de los Descalzos, el estanque de Santa Beatriz, el Paseo de Aguas o el callejón de Matamandinga ${ }^{1}$.

Al llegar 1860, superados sus años de cronista local, la Marina peruana cuenta con Palma entre sus funcionarios administrativos responsables; en sus escenarios, las salas limeñas habían visto representar cuatro de sus dramas e igual número de sus comedias; la imprenta de J. M. Masías había publicado un voluminoso volumen con sus poesías juveniles; la masonería de la capital y la del Callao lo habían incorporado a las logias «Concordia Universal» y «Virtud y Unión» y, acreditando su juvenil entusiasmo, se compromete en un atentado nada menos que contra la vida del presidente de la república. Palma era, pues, un personaje vigente.

Distinto fue el caso del autor de Pájinas libres. Formaba parte de una familia de diligentes funcionarios públicos, pues en la época del virrey Pezuela su abuelo había sido Superintendente General de Hacienda y en años republicanos su padre, juez civil y prestigioso abogado en Arequipa. Instalada la familia González Prada en Lima, disuelta que fuera la Confederación Perú-

l Sobre los primeros treinta años de vida de Palma es imprescindible recurrir al exhaustivo trabajo de Oswaldo Holguín Callo, Tiempos de infancia y bohemia. Ricardo Palma (1833-1860), en el que se describe con minuciosidad la ciudad de Lima y la vida que en ella imperaba. 
Boliviana, el pater familias, don Francisco González de Prada, bien asentado en el ámbito político más cercano al presidente Echenique, asciende con rapidez y llega a ser ministro de estado y vicepresidente de la república: precisamente por eso, después de la batalla de La Palma (1855) en que las fuerzas revolucionarias encabezadas por Castilla derrotan a las gubernamentales, debe partir, con toda la familia, por un par de años al exilio en Chile. Nuevamente en Lima, es elegido Decano del Colegio de Abogados y, prescindiendo de cualquier rivalidad política, Castilla lo designa alcalde de la capital peruana. Los González Prada constituían, pues, una familia social y políticamente prominente ${ }^{2}$.

Durante su juventud, los dos escritores estuvieron vinculados al Convictorio de San Carlos; sin embargo, la destrucción de sus archivos durante la Guerra del Pacífico no ha permitido analizar en detalle su rendimiento. Palma debe haber estado inscrito entre 1849 y 1850/5 I; Prada, entre 1860 y 1863/64. Ninguno de los dos escritores llegó a completar sus estudios: Holguín, quien ha dirimido en la polémica sobre si Palma fue o no carolino, cree que don Ricardo Palma fue atraído por el periodismo (1994); Sánchez (1977), por su parte, estima que Prada se retiró dada su aversión al latín, pese a que dominaba el idioma.

\section{Participando en dos guerras}

La primera actividad que Palma y González Prada pudieron haber compartido -aunque en niveles diferentes-fue el combate

2 Luis Alberto Sánchez ha estudiado la figura de González Prada en dos libros fundamentales: Don Manuel (Lima, F. y Ed. Rosay, 1930, p. 266), reeditado con algunas adiciones por la Universidad Nacional Mayor de San Marcos en 1960 (p. 260) y Nuestras vidas son los ríos. Historia y leyenda de los González Prada (Lima, Universidad Nacional Mayor de San Marcos, 1877, p. 405). Sánchez pudo disponer del archivo de la familia González Prada que, en vida, donó a la Biblioteca Nacional peruana. Por ello, sus aportes al conocimiento de vida y obra de González Prada son inmejorables. 
del 2 de mayo de 1866 durante la guerra con España: Prada, integrando el batallón de estudiantes ${ }^{3}$; Palma, en la Secretaría de Guerra y Marina, en posición cercana al Estado Mayor. Ambos, por cierto, celebraron el triunfo que, de otra parte, para ellos se mostraba enlutado por la trágica muerte de José Gálvez, uno de los líderes del liberalismo peruano de entonces, por quien ambos tenían viva simpatía.

Recién por aquel tiempo, cuando ya habían transcurrido 19 años desde que Palma publicara su primera composición en El Comercio, Prada se da a conocer como poeta publicando en el mismo diario, el 18 de setiembre de 1867, una letrilla que suscribe como «Manuel G. P.». Prada, pues, no ingresa al mundo de lo literario con la precocidad de otros. Pero mientras Palma ya había sufrido dos años de destierro en Valparaíso y Santiago de Chile y había conocido Francia y otros países europeos en extenso periplo rumbo a un frustrado puesto consular en Brasil y se había enrolado al pequeño grupo de fuerzas revolucionarias que el coronel Balta había levantado en el norte del país y que habría de triunfar en 1867, Prada había permanecido en la capital con viajes cada vez más frecuentes y permanencias más prolongadas en Tútumo, predio agrícola de mediana extensión del cual la familia era propietaria, ubicado en el valle de Mala, a 100 kilómetros al sur de Lima.

3 Desconozco quién ha aportado este dato que se viene repitiendo hace casi un siglo. Sin embargo, un investigador tan extraordinariamente acucioso como Zanutelli observa que en el «Índice alfabético de los vencedores del 2 de mayo de 1866 y de los vencedores de Abtao en el combate naval del 7 de febrero del mismo año» que se conserva en el Archivo Histórico Militar del Perú no figura don Manuel ni como Gonzalez ni como Prada (2003, p. 42). 


\section{Una relación amistosa}

Los primeros contactos literarios entre ambos escritores deben haber ocurrido hacia 1871. Por aquellos años, Palma gozaba de importancia política como senador por Loreto y secretario personal del presidente Balta y las prensas locales estaban muy interesadas en publicar sus ya famosas Tradiciones. ${ }^{4}$ Mientras tanto, Prada estaba en Tútumo, donde cultivaba yuca y desarrollaba algunos ensayos semiindustriales con el propósito de producir almidón (Gonzales Prada, 1985, pp. 127-134) . No hay duda de que su retiro campestre le daba tiempo para escribir pues a partir de 1867 aparecieron -siempre con seudónimo- sus primeros artículos en el diario limeño El Nacional y sus primeros rondós y triolets. Más aún, en ese año, cuando José Domingo Cortés prepara su antología con obras de los más destacados poetas peruanos de entonces, se refiere a este joven escritor términos de buen observador:

Ha escrito para dar pábulo a su corazón, sin ir en busca de la aura popular, sin lanzar sus obras a la publicidad, tras un aplauso o una felicitación. Esto esplica (sic) suficientemente la parsimonia con que ha dado a la prensa sus producciones. (1871, p. 323)

Es decir, hasta un antologista como Cortés advertía la cautela con que González Prada se acercaba a la prensa con lo que

4 Fernando Flores-Zúñiga, Ricardo Palma y la tribuna parlamentaria. El tradicionista senador (1868-1873). Lima, Fondo Editorial del Congreso del Perú, 2019, p. 438 .

5 En algún momento entre 1870 y 1879, Prada preparó una de lo que llamó sus memorias titulada «Algo sobre el almidón y sus derivados». Este trabajo permaneció como manuscrito hasta que su hijo Alfredo lo publicó en El tonel de Diógenes. Posteriormente se reprodujo en Manuel González Prada, Obras. Tomo I, vol. 2, pp. 127-134. (Lima, Ediciones Copé, 1985.) Se dice-se desconoce el origen de la aseveración- que González Prada llegó, en algún momento, a ser el principal proveedor de almidón para la capital. 
venía escribiendo en Lima o en la soledad de Mala, a la inversa de los románticos peruanos, los integrantes de la bohemia que tan bien describe Palma, que hacían públicas sus obras antes de haber cumplido los veinte años. Prada da publicidad a sus primeros poemas cuando ya es algo mayor ${ }^{6}$.

El mismo año 1871 es testigo de un segundo contacto literario entre ambos literatos. En El Correo del Perú, excelente revista cultural limeña aparecida entre 1871 y 1878, que dirigía Trinidad M. Pérez, se recogen textos de los dos escritores: de Prada, diez poemas, cinco de ellos rotulados como rondeles, todos suscritos como «M. G. Prada»; de Palma, más de ochenta: tradiciones, reseñas a trabajos peruanos y extranjeros, traducciones, artículos, etc. El editor evidentemente discrimina: los poemas de González Prada no le merecen comentario alguno; a la inversa, los de Palma se anuncian con cierto alborozo adelantando que el autor se ha comprometido a colaborar con frecuencia y detallando a qué serie pertenecen las tradiciones que habrán de publicarse. Es evidente que los dos escritores merecen un trato diferente del editor, lo cual podría explicarse por el hecho de que tanto Trinidad Pérez como su hermano Isidro Mariano habían formado parte, con Palma, de la bohemia literaria de 1848; mientras tanto, Prada era un poeta joven que aparecía cultivando una poesía evidentemente distinta.

Otra revista cultural, aunque de menor duración, La Bella Limeña, que dirigía Abel de la E. Delgado, también publica obras de Palma y Prada - por supuesto, una tradición y un poema, respectivamente- y en algún momento los menciona entre

6 Palma es un buen ejemplo de ello: cuando acababa de cumplir 15 años de edad, El Comercio de Lima publica su primer poema; antes de cumplir los 18 ya había estrenado cuatro dramas y publicado cuando menos uno (se ignora si alguno de los otros tres fue publicado y luego destruido por el autor). 
sus colaboradores de mayor importancia ${ }^{7}$. Y una revisión más detenida de otras publicaciones de aquella década permitiría descubrir aportes adicionales de ambos escritores.

Por último, en 1874, al publicar los Anales de la Sección de Literatura, el Club Literario de Lima menciona, en orden alfabético, entre sus 30 integrantes a los señores Manuel González Prada y Ricardo Palma ${ }^{8}$. Con el tiempo, Prada llegaría a ser vicepresidente de la Sección.

Es evidente que por aquellos años ya existía una relación frecuente entre Palma y Prada, la misma que se mantuvo hasta varios años después del final de la Guerra del Pacífico, en la cual hicieron causa común con su país: don Ricardo en la batalla de Miraflores y don Manuel como capitán de la primera compañía del batallón $\mathrm{N}^{\circ} 50$ asignado a la defensa del reducto establecido en el cerro El Pino 9 . Los dos escritores sintieron en carne propia los efectos de la ocupación de Lima: no es posible olvidar las gestiones de Palma como subdirector honorario de la Biblioteca que condujeron a su apresamiento y casi a su deportación a Chile ni el enclaustramiento al cual Prada se sometió durante aquellos años en su casa de la calle de La Merced en el centro de la capital.

A fines de 1885, en medio de la crisis causada por la guerra, González Prada aborda a Palma y le manifiesta su interés en conocer las traducciones que este había hecho de algunas poesías de Heine, puesto que debía ofrecer una conferencia

7 En La Bella Limeña, año I, Núm. 5, p. 1. Lima, 5 de mayo de 1872.

8 Club Literario de Lima, Anales de la Sección de Literatura. Primer año. 18731874. P. (1).

9 Es indudable que Palma participó en la batalla de Miraflores (15 de enero de 1881) y junto a cientos de peruanos luego tuvo que caminar hasta Lima; la información sobre la participación de González Prada en Zanutelli (2003), La saga de los González Prada, pp. 41-42. 
sobre el autor alemán en El Ateneo de Lima ${ }^{10}$. El tono de la petición, formulada posiblemente por escrito, debe haber sido tan amable que Palma acoge el pedido y le envía las traducciones con un nota tan cordial que conviene recordar (Palma, 2005, pp. 386-387; el resaltado es nuestro):

Señor don Manuel González Prada.

\section{Mi buen amigo:}

Con motivo de la conferencia que, sobre Enrique Heine y sus obras, se propone usted dar en el Ateneo de Lima, me ha manifestado empeño en conocer las pocas traducciones del tan notable poeta alemán que hice, allá en los tiempos en que mi pluma era tributaria de las musas. Pídeme usted, y hácelo con tan afectuosos modos, que no me deja tiempo para la excusa.

Le remito, pues, mis traducciones de Heine que distan mucho en galanura, lijereza (sic) y corrección de forma, de las seis que conozco de Eulogio Florentino Sanz [...]

Decir a usted que Heine llegó a ser uno de mis poetas favoritos sería redundancia. No se emplea el tiempo en traducir a un autor por quien no se siente uno encariñado. Juzgar a Heine, y a los que hemos intentado darlo a conocer en los pueblos donde es familiar la rica habla de Castilla, cumple al claro talento y buen gusto literario que en usted se complace en reconocer su amigo y compañero afectísimo.

Ricardo Palma.

Lima, Diciembre 25 de 1885.

I0 Palma, que no hablaba alemán, tradujo al español a Heine (1797-1856) que ya había sido traducido al francés. Sus traducciones habían aparecido en algunas revistas limeñas hasta que las recopiló y publicó como Enrique Heine. Traducciones (Lima, Imprenta del Teatro, 1886). Por lo tanto, cuando González Prada se las solicita, el pequeño libro (p. 23) con las traducciones aún no estaba impreso. La conferencia a la cual alude Prada es la conocida como «Conferencia en el Ateneo de Lima» que se menciona más adelante, y que con múltiples correcciones es el primero de los textos que conforman Pájinas libres. 
El pedido de González Prada, pues, había sido formulado en tan afectuosos modos que Palma no podía excusar su atención; a la inversa, don Ricardo se complace en reconocer que era amigo y compañero afectísimo de González Prada quien estaba dotado de un claro talento y buen gusto literario. ¿Cabía entre ambos una relación mejor o más estrecha? En nuestro entender, pues, a fines de 1885 había entre ambos escritores una relación inmejorable.

\section{El inicio de una confrontación}

En 1883, la Imprenta del Universo de Carlos Prince había publicado las seis series de Tradiciones peruanas de Palma y, en Nueva York, la Imprenta de Las Novedades la docena de textos que forman parte de El demonio de los Andes. En los años que siguieron nada distrajo a Palma de su labor de director de la Biblioteca Nacional y, con excepción del folleto que contiene sus ya antiguas traducciones de Enrique Heine, no ofrecerá nada nuevo hasta 1889.

Por otra parte, el cercano recuerdo de la humillante derrota en la Guerra del Pacífico, el interés en no tener que presenciar nuevamente motines ni rebeliones ni guerras internas como las que habían abundado en el Perú republicano y que tantos males le habían ocasionado ${ }^{11}$ y la convicción de que era necesario modificar la orientación del país tenía a los jóvenes y a los no tan jóvenes interesados en una renovación, así no pudieran precisar qué es lo que pretendían cambiar. Esta sensación, que era común a todos los grupos sociales, políticos y económicos, también lo era dentro del ámbito que ahora interesa, el cultural y el literario, y prueba de ello es que los integrantes del Club Literario, en sesión de 11 de noviembre de 1885, acuerdan

11 Casualmente, habían pasado solo tres semanas desde que el 3 de diciembre había concluido la que habían sostenido los generales Miguel Iglesias y Andrés Avelino Cáceres. 
modificar su estructura, redefinir sus propósitos y hasta cambiar de nombre: el Club Literario pasa a ser el Ateneo de Lima. Prueba de la consideración que tenían sus integrantes por Prada es que en su directiva inicial aparece como $2^{\circ}$ vicepresidente, detrás de Eugenio Larrabure y Unánue y Ricardo Rossel.

El 30 de enero de 1886 tiene lugar la ceremonia de instalación de la Sección de Literatura y Bellas Artes del flamante Ateneo y el discurso de instalación corre a cargo de Prada ${ }^{12}$. Se trata del discurso que, una década más tarde, habrá de encabezar Pájinas libres.

Varios meses antes (abril de 1885) había aparecido una publicación de carácter político y literario que acreditaba el interés de un grupo de políticos, escritores y periodistas, en su mayoría jóvenes, por cambiar el país. Era La Revista Social. Como el éxito de este semanario fue grande, después de 18 meses de circulación sus promotores decidieron constituirse como una alternativa juvenil y rebelde contra el maduro y prudente Ateneo de Lima. Y es así como en octubre de 1886 se inaugura el Círculo Literario.

Tanto la publicación de La Revista Social cuanto la creación del Círculo Literario eran ideas loables. Pero debe notarse que uno de los más importantes redactores de La Revista Social y futuro primer presidente del Círculo fue Luis Enrique Márquez, un antiguo y radical enemigo político de Palma. Y fue precisamente

12 La versión más conocida de este discurso proviene de la edición de Luis Alberto Sánchez, Pájinas (sic) libres (Lima, Editorial PTCM, 1946), el primer volumen de una pretendida edición de las obras completas de González Prada. En la «Introducción» de esa edición, Sánchez declara que la suya es la tercera edición y solo menciona la preparada por el propio Prada en Francia en 1894 y la de Rufino Blanco Fombona (1914). La versión original del discurso apareció en El Ateneo de Lima, tomo I, pp. 29-47 (Lima, Imp. Del Teatro, 1886). Quienes pretendan estudiar seriamente la prosa de don Manuel no pueden dejar de tenerla presente. 
en su casa donde se establece el Círculo Literario. Lo que merece un comentario especial (Varillas, 2014) ${ }^{13}$.

Luis E. Márquez (1846-1886), hermano menor del poeta Arnaldo Márquez, fue un calificado periodista y versificador satírico de primer nivel ${ }^{14}$. En 1871, cuando era jefe de informaciones locales en el diario El Nacional, se organiza la campaña presidencial de Manuel Pardo y, en su apoyo, Márquez edita durante algún tiempo La Sabatina, agresivo semanario político y humorístico ${ }^{15}$. Tres meses después de la asunción de Pardo al mando supremo, luego del vil asesinato del presidente coronel Balta durante el motín de los hermanos Gutiérrez, aparece El Cascabel con el propósito de atacar a Pardo y al Partido Civil ${ }^{16}$; enterado Márquez de que Palma era uno de

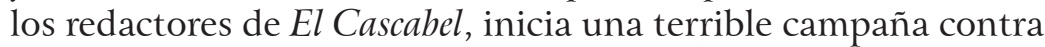
don Ricardo, quien meses atrás acababa de publicar su Primera Serie de Tradiciones. En cuatro de los quince números de La Sabatina aparecidos en el segundo semestre de 1872, Márquez centra sus ataques en Palma atribuyéndole ser 'tronchista' y 'capitulero'17. Lo penoso de la actitud de Márquez es que en

13 Sobre el asunto presenté hace algunos años, en la decimotercera jornada del Encuentro Internacional Re-visión de las Tradiciones, la ponencia titulada «La Sabatina, Luis Enrique Márquez y Ricardo Palma». Posteriormente se publicó, con algunas adiciones, con el título de «Un desconocido incidente periodístico de Ricardo Palma» en Aula Palma XIII. pp. (357)-359, Lima, Instituto Ricardo Palma, 2014.

14 Luis E. Márquez había estudiado en Valparaíso y Nueva York y, posiblemente en Bélgica e Italia. Incorporado al servicio diplomático peruano, se desempeñó como cónsul en Panamá, Valparaíso e Iquique antes de ser designado Oficial Mayor del Ministerio de Relaciones Exteriores.

15 La Sabatina fue un semanario político publicado en Lima del cual aparecen 40 números entre el 23 de junio de 1871 y el 5 de abril de 1873 . Con excepción de los números correspondientes a 1872, su aparición fue relativamente puntual.

16 De El Cascabel aparecieron 68 números entre el 26 de octubre de 1872 y el 5 de setiembre de 1874 .

17 El Diccionario de Peruanismos (Lima, Compañía de Minas Buenaventura y Academia Peruana de la Lengua, 2016) establece que 'tronchista' es «quien gestiona provecho para sí sin esfuerzo o ilícitamente» y 'capitulero', «quien busca votos con intrigas». 
buena parte de los ataques contra Palma se refiere en términos irrepetibles contra la madre de Palma y a la aparentemente ascendencia mulata del tradicionista:

Prieta la cara y más prieta

el alma que Dios le dio,

este prieto comenzó

por dedicarse a poeta $[\ldots]$

Fue más tarde secretario

tierno, sumiso, indulgente

y una ardilla como ajente (sic)

de cualquier grande empresario ${ }^{18}$.

Y en todo momento a propósito de Palma reitera categorías raciales:

Un negrito lanzó un grito

a la puerta del infierno

y salió el diablo y le dijo

iaquí no gritan los negros!

Los textos anteriores y otros cuya transcripción deliberadamente omito no prestigian a Márquez, pero tienen como propósito mostrar cómo desde 1872 entre Palma y Márquez difícilmente cabía reconciliación alguna ${ }^{19}$.

18 Este texto y el siguiente están tomados de mi artículo titulado «Un desconocido incidente periodístico de Ricardo Palma» en Aula Palma XIII, pp. 357-380, Lima, Instituto Ricardo Palma, 2014.

19 En un trabajo mío antes citado (Varillas, 1992), insinúo que, dada la exacerbación de la confrontación, el nivel de vulgaridad de los ataques y la forma en que ellos desaparecen a principios de 1873, era posible que entre los propietarios de La Sabatina y El Cascabel -que no eran Palma ni Márquez- se haya tomado en cuenta que estando Pardo consolidado en la presidencia el intercambio de insultos era inconducente. 


\section{Una situación que se complica}

Volvamos al tema que ahora nos convoca. Después de habérsele asignado el discurso de orden en la ceremonia de instalación de la Sección de Lengua y Literatura del Ateneo de Lima (30 de enero de 1886) es evidente que González Prada pasaba a disfrutar una posición de preeminencia dentro del mundo literario peruano. A ese reconocimiento debe añadirse uno adicional: en el domicilio de Luis Enrique Márquez se reúnen algunos escritores y periodistas con los redactores de La Revista Social y acuerdan la fundación del Círculo Literario y para presidir su primera junta directiva eligen a Márquez y como vicepresidente a González Prada (octubre de 1886).

Como ya se ha indicado, por entonces Palma estaba contraído a su labor de director de la Biblioteca pero, adicionalmente, estaba enfrascado, tanto a título personal cuanto como masón de alto grado, en la campaña destinada a lograr la expulsión de los jesuitas del Perú ${ }^{20}$. La campaña cubre la mitad del año 1886, entre marzo y octubre. Y ese momento fue la oportunidad para que don Manuel se desvinculara del Ateneo y se concentrara en La Revista Social y el Círculo Literario.

A partir de 1887, diversos hechos comienzan a llevarse a cabo con gran rapidez: a mediados de año fallece la madre de don Manuel, quien se había opuesto a su matrimonio; la salud de Márquez comienza a deteriorarse; en octubre de 1887 Prada

20 Como se sabe, los jesuitas, que habían sido expulsados del imperio español por el rey Carlos III en 1767, habían sido readmitidos al Perú durante el gobierno del presidente Balta (1871); sin embargo, a raíz de la publicación por el p. Ricardo Cappa S. J. de un Compendio de Historia del Perú de 219 pp. (Lima, 1886) se desató una campaña a nivel nacional destinada a su expulsión del Perú. La campaña se inició en marzo de 1886, llegó a su punto culminante en el mes de julio y los últimos jesuitas abandonaron el país en octubre del mismo año. 
contrae enlace con Adriana Verneuil, veinte años menor que él y que lo sobrevivirá; mientras Prada se encontraba gozando de los primeros días de su matrimonio, Márquez renuncia por razones de salud a la presidencia del Circulo Literario y don Manuel, quien no se encontraba en la asamblea, es elegido presidente por aclamación; en abril de 1888 fallece Márquez y don Manuel despide sus restos con un breve y enérgico discurso; en el Teatro Olimpo, da comienzo a su actitud desafiante con un discurso contra la influencia española en la literatura peruana ${ }^{21}$; el 29 de julio de 1888 pronuncia, ante el presidente de la república y autoridades de todo nivel, su famoso Discurso en el Politeama ${ }^{22}$. Ya la suerte estaba echada y no cabía retractación alguna.

\section{La reacción de Ricardo Palma}

Por cierto, Palma se sintió aludido por los discursos de Prada, pero su epistolario registra solo tres ocasiones en que se ocupa de la situación. En la primera, refiere lo ocurrido a su amigo, el poeta e historiador mejicano Francisco Sosa:

En estos días he estado muy agitado. Una asociación de muchachos dio una función en el teatro, en la que hubo discursos muy insolentes contra los Académicos, contra España y contra los hombres que peinan canas culpando a estos de todos los males del Perú -Los jóvenes a la obra

21 A este discurso, que luego aparece en Pájinas libres, pertenece una de las más conocidas frases de Prada: «...en la prosa, reina siempre la mala tradición, ese monstruo enjendrado por las falsificaciones agridulcetes de la historia i la caricatura microscópica de la novela». El texto corresponde al Discurso en el Teatro Olimpo pronunciado el 29 de julio de 1888 y González Prada lo incluye en Pájinas libres (en Obras, Tomo I, vol. 1, p. 65).

22 En este discurso, también recogido en Pájinas libres, Prada pronunció una de las frases más famosas y radicales de la oratoria peruana: "Los viejos a la tumba, los jóvenes a la obra”. Pertenece a su Discurso en el Politeama. En: Obras, Tomo I, vol. 1, p. 90 . 
y los viejos a la tumba- fue el tema sobre el que disertó largo el Presidente de esa Sociedad, que es un caballero de 44 años y no falto de talento y de ilustración. Hablando de mis tradiciones, pues todo su discurso fue personalísimo contra Roca, Lavalle, Cisneros y demás académicos, dijo que las tradiciones eran una caricatura de la Historia. Desatendiéndome de lo que a mi tañía, publiqué un largo artículo desafiando al señor González Prada a sostener sus doctrinas en una... [trunca]. (Palma, 2005, 386-387)23.

Años después, cuando la tensión había amainado, Palma informa de ella a don Miguel de Unamuno:

González Prada (radical) y yo (liberal) vivimos alejados y sin cambiar saludo desde 1890. En un discurso lanzó esta frase de adulación para los muchachos inquietos -los viejos a la tumba y los jóvenes a la obra. Yo, que aún tenía nervios irritables y sangre fosfórica, pues peinaba 57 febreros, refuté su frase en un artículo al que don Manuel no quiso contestar, encomendando a la jauría de sus devotos alborotadores que amasasen el lodo de las calles y me lo echasen a la cara. Hoy González Prada se aproxima a los 60 diciembres, y no sé si aun sostendrá aquel $s u$ aforismo (Palma, 2006, pp. 12-13) 24.

Y por último, ya en las últimas cursadas a Unamuno alude a Prada como «hombre roído por la envidia» (ibíd., p. 291 $)^{25}$. Aparece con claridad que después de los ataques sufridos, en

23 Carta de Ricardo Palma a Francisco Sosa, de 28 de noviembre de 1889. La carta no corresponde a 1889 sino a 1888. Es difícil no creer que hay un error del editor de este Epistolario de Palma, que puede provenir de la fuente que ha seguido el Epistolario publicado en 1949 por la Editorial Cultura Antártica en el cual hay varios errores de este tipo. Los hechos a los que se refiere Palma ocurren en 1888: en 1889, ni Palma ni González Prada se ocupaban del asunto.

24 Carta de Ricardo Palma a Miguel de Unamuno, de 19 de diciembre de 1905.

25 Carta de Ricardo Palma a Miguel de Unamuno, de 16 de febrero de 1913. 
los cuales no había participado don Manuel, Palma se limita a su labor de bibliotecario hasta llegar a la ancianidad.

\section{La insistencia de González Prada}

Una lectura cuidadosa de la obra de González Prada demostraría que en los años siguientes don Manuel eludió atacar a Palma aunque sus ataques al virreinato peruano, a lo nociva que era la influencia de la literatura española en la peruana y la Academia correspondiente de la Real Española en el Perú estaban enrumbados hacia el tradicionista.

Pero en privado la situación era diferente. De por aquellos años es el «Yambo 2», que forma parte del primero de los cuadernos manuscritos dejados por Prada, Letrillas y El canto del otro siglo. Alfredo González Prada y Luis Alberto Sánchez, heredero el primero y legatario el segundo, tuvieron en su poder el texto, pero optaron por no publicarlo en espera de una edición crítica que nunca apareció. En 1977, Sánchez lo publicó calificándolo como texto «hasta hoy merecidamente ignorado». Su siguiente edición, dentro del último volumen de las Obras de Prada no le merece comentario alguno. Se presentan dos de las trece estrofas de la composición, ambas se refieren a la obra y no al autor (Sánchez, 1977, pp. 113-114):

Embutidor imbécil de refranes

En prosa estrafalaria,

Prueba en el estilo y en los planes

Tu fatuidad palmaria. [...]

Treinta años ha de parir no dejas

Y ¿qué son tus abortos?

Cuentecillos de niñas y de viejas

Tan malos como cortos. 
González Prada tuvo el buen gusto de no publicar esta composición; quien escribe estas líneas tiene la impresión de que nunca hubiera autorizado que llegara a difundirse.

\section{Recapitulación}

Se ha tratado de historiar la contienda que hubo entre Palma y González Prada hasta antes de 1890 a base de hechos y de los testimonios de los dos escritores. Se ha prescindido deliberadamente de otras propuestas ya formuladas, prescindiendo de la jerarquía del crítico que la hubiera formulado, puesto que estimamos que son susceptibles de revisión.

Resumiendo, creemos que la confrontación entre estos dos escritores, se produjo por tres razones:

1. Se trató de una confrontación generacional entre la Generación Romántica de la cual, después de la muerte de Corpancho (1863), Palma era el líder indiscutido y exitoso a nivel internacional, y la Generación Ecléctica, cuyos integrantes se aglutinaron alrededor de González Prada una vez que se pudo advertir que este, a base de una oratoria vibrante, podía encabezar la reacción de la que el Perú estaba ansioso luego de la Guerra del Pacífico.

Tal como lo ha estudiado Ortega y Gasset en El tema de nuestro tiempo (1923) cada generación puede tener un carácter cumulativo (cuando sus integrantes sienten que entre lo recibido de la generación anterior y lo que les es propio existe una suficiente homogeneidad) o un carácter polémico (cuando entre lo recibido y lo propio hay una total heterogeneidad): estas son generaciones 
de combate. Ese carácter tuvo la Generación Ecléctica: mientras la experiencia generacional de los Románticos fue el triunfo en la guerra con España (1863-1866) la de los Eclécticos fue la formidable derrota de la guerra del Pacífico (1879-1884).

Por lo tanto, la Generación Ecléctica estaba destinada a romper los lazos que la vinculaban a la generación anterior, la Romántica.

2. La presencia de un hombre como Luis E. Márquez hace posible que se reúna alrededor suyo en La Revista Social y en el Círculo Literario un grupo de políticos, escritores y periodistas emergentes que en ese momento vivían en un ambiente de absoluta insatisfacción. Cuando Márquez fallece (abril de 1888), el grupo encuentra un sucesor idóneo en Prada (que ya era vicepresidente del Círculo) y después del exitosísimo Discurso del Politeama (julio de 1888) fue tan evidente que don Manuel era el líder que buscaban que de inmediato lo eligen Presidente Vitalicio del Círculo (agosto de 1888).

Siguiendo a Márquez, el Círculo Literario y sus integrantes la emprenden contra Palma y cuando Márquez fallece, González Prada repara en que era a él a quien le tocaba capitanear la campaña contra Palma y los románticos (es decir, contra los mayores que los habían conducido a la derrota en la guerra).

3. Ricardo Palma había sido la cabeza visible de la literatura peruana desde el inicio de la segunda mitad del siglo XIX. Hacia 1888, Palma, que siempre se quejó de su salud, contaba con 55 años de edad; Prada quien había recibido parte de su formación en Valparaíso, tenía 44 años y llevaba casi una década de trabajo en 
el campo. Desde una perspectiva generacional, Palma estaba saliendo del grupo que 'estaba en el poder' Prada estaba ingresando a él.

Creemos que así es posible explicar las razones por las cuales ambos escritores, los más importantes del siglo XIX peruano, terminaron enfrascados en un violento enfrentamiento que no benefició a ninguno de ellos.

\section{Referencias bibliográficas}

Cornejo Polar, A. (1982). Historia de la literatura en el Perú republicano. Tomo VIII de la Historia del Perú Republicano, pp.11-188. Lima: Editorial Juan Mejía Baca.

Cortés, J. D. (1871). Parnaso peruano. Valparaíso: Imprenta Albión de Cox y Taylor.

Delgado, W. (1980). Historia de la literatura republicana. Lima: Ediciones Rikchay Perú.

García Calderón, V. (1938). Los románticos. Tomo № 8 de la Biblioteca de Cultura Peruana. París: Desclée de Brouwer.

González Prada, M. (1985). Obras. Tomo I. Vol. 2. Lima: Ediciones Copé.

Holguín Callo, O. (1994). Tiempos de infancia y bohemia. Ricardo Palma (1933-1860). Lima: Pontificia Universidad Católica del Perú.

. (2001). Páginas sobre Palma. Lima: Universidad Ricardo

Palma.

Moncloa y Covarrubias, M. (1902). Los bohemios de 1886. Lima: G. Stotle. 
Nieto Vélez, A. (1978). Historia del Colegio de la Inmaculada. Lima: Editora Turística Perú S.C.R.L.

Palma, R. (2005). Epistolario. Vol. 1. Lima: Editorial Universitaria de la Universidad Ricardo Palma.

. (2006). Epistolario. Vol. 3. Lima: Editorial Universitaria de la Universidad Ricardo Palma.

Riva Agüero y Osma, J. de la. (1905). Carácter de la literatura del Perú Independiente.

Sánchez, L. A. (1977). Nuestras vidas son los ríos... Historia y leyenda de los González Prada. Lima: Universidad Nacional Mayor de San Marcos.

Tamayo Vargas, A. (1992). Literatura peruana. Lima: PEISA.

Varillas Montenegro, A. (1992). La literatura peruana del siglo XIX. Periodificación y caracterización. Periodificación y caracterización. Lima: Pontificia Universidad Católica del Perú.

Varillas Montenegro, A. (2014). «Un desconocido incidente periodístico de Ricardo Palma». En Aula Palma. No 13. Lima: Universidad Ricardo Palma.

Zanutelli Rosas, M. (2003). La saga de los González Prada. Lima: Editorial Universitaria de la Universidad Ricardo Palma.

Recibido el 26 de agosto de 2020 Aceptado el 28 de septiembre de 2020 